\title{
A METRIC APPROACH TOWARD POINT PROCESS DIVERGENCE
}

\author{
Sohan Seth, Austin J. Brockmeier, José C. Príncipe \\ Electrical and Computer Engineering \\ University of Florida, Gainesville, Florida \\ \{sohan,ajbrockmeier,principe\}@cnel.ufl.edu
}

\begin{abstract}
Estimating divergence between two point processes, i.e. probability laws on the space of spike trains, is an essential tool in many computational neuroscience applications, such as change detection and neural coding. However, the problem of estimating divergence, although well studied in the Euclidean space, has seldom been addressed in a more general setting. Since the space of spike trains can be viewed as a metric space, we address the problem of estimating JensenShannon divergence in a metric space using a nearest neighbor based approach. We empirically demonstrate the validity of the proposed estimator, and compare it against other available methods in the context of two-sample problem.
\end{abstract}

Index Terms - Divergence, metric space, point process, nearest neighbor, hypothesis testing

\section{INTRODUCTION}

In the neuroscience literature, it is a well accepted fact that neurons communicate via sequence of action potentials or spikes. This sequence is formally known as a neural spike train, and the probability law over the set of spike trains is formally known as a point process. Many computational neuroscience applications such as change detection, can be framed as a two-sample problem, that requires evaluating a suitable divergence measure between two point processes; where a divergence measure is defined as a non-negative statistic of two probability laws that attains zero value if and only if the probability laws are the same. However, estimating divergence between two point processes is not trivial since the space of spike trains lacks basic algebraic and topological structures.

Recently, [5] and [7] have proposed two conceptually different approaches for assessing divergence between two point processes: [5] has addressed the problem of estimating Hellinger distance between two point processes, whereas [7] has extended the Kolmogorov-Smirnov and the Cramér-vonMises tests to the space of spike trains. Both these approaches rely on representing the space of spike trains as a direct sum

The work has been partially supported by NSF grant ECCS-0856441 and DARPA grant N66001-10-C-2008. The authors thank Dr. Il Park for valuable discussions at an early stage of this project. of Euclidean spaces, called strata, and evaluating the divergence separately in each stratum. Although theoretically sound and computationally efficient, these approaches have a serious drawback; they do not allow any interaction between two spike trains from two different strata. Therefore, these approaches suffer in estimation since the strata are created based on the spike counts, and given a finite number of spike trains, it becomes hard to populate each stratum sufficiently, especially when the underlying point process has a relatively flat count distribution. This issue restricts the applicability of these approaches. In this paper, we explore an alternate method of estimating divergence that do not require stratification.

In an Euclidean space, the problem of estimating divergence can be addressed in several ways, e.g. using the empirical cumulative distribution function or the empirical RandonNikodym derivative $[2,8,10]$. These approaches, however, cannot be readily applied to a more general space. The former approach requires the underlying space to have an order. On the other hand, the latter approach can be materialized in several ways including using a nearest neighbor based approach [10]. We aim at extending this approach since, conceptually, it only requires the underlying space to have a metric. Since, recent research in the field of neuroscience have shown that the space of spike trains can be treated as a metric space [9], it allows the proposed estimator to be used in the context of estimating divergence between two point processes.

The rest of the paper is organized as follows. In section 2 we describe the difficulties of extending the traditional methods of estimating divergence to a more general space, and then propose a method of estimating Jensen-Shannon divergence in a metric space. We choose this divergence since it is widely used as a metric in the space of probability measures [4]. In section 3 we discuss the effect of the choice of metric on the convergence of the estimated divergence value, and present three simulated hypothesis testing experiments to demonstrate the validity and the drawbacks of the current view of estimating divergence. In section 4 we conclude the paper with some formal discussion on the pros and cons of the proposed method, and some guidelines of future work. 


\section{DIVERGENCE: DEFINITION AND ESTIMATION}

Let $\mathcal{X}$ be a metric space equipped with the metric $\delta$, and let $X$ and $Y$ be two random variables that take values in $\mathcal{X}$. Assume that these random variables follow the probability laws $\mathbb{P}$ and $\mathbb{Q}$ respectively i.e. $X \sim \mathbb{P}$ and $Y \sim \mathbb{Q}$. Then the KullbackLeibler (KL) divergence is defined as

$$
\mathbb{D}_{K L}(\mathbb{P}, \mathbb{Q})=\int \log \left(\frac{\mathrm{d} \mathbb{P} / \mathrm{d} \mu}{\mathrm{d} \mathbb{Q} / \mathrm{d} \mu}\right) \frac{\mathrm{d} \mathbb{P}}{\mathrm{d} \mu} \mathrm{d} \mu=\mathbf{E}_{X} \log \frac{\mathrm{d} \mathbb{P}}{\mathrm{d} \mathbb{Q}}
$$

where $\mu$ is a probability law on $\mathcal{X}$ such that $\mathbb{P} \ll \mathbb{Q} \ll \mu$. It is evident that the estimation of KL-divergence relies on the estimation of the individual Radon-Nikodym derivatives $\mathrm{d} \mathbb{P} / \mathrm{d} \mu$ and $\mathrm{d} \mathbb{Q} / \mathrm{d} \mu$, or just the Radon-Nikodym derivative $\mathrm{d} \mathbb{P} / \mathrm{d} \mathbb{Q}$. Moreover,

$$
\hat{\mathbb{D}}_{K L}(\mathbb{P}, \mathbb{Q})=\frac{1}{n} \sum_{i=1}^{n} \log \frac{\widehat{\mathrm{dP}}}{\mathrm{d} \mathbb{Q}}\left(x_{i}\right)
$$

where $\left\{x_{i}\right\}_{i=1}^{n}$ and $\left\{y_{i}\right\}_{i=1}^{m}$ are samples from $X$ and $Y$ respectively, and $\hat{\bullet}$ denotes the sample estimate.

In $\mathbb{R}^{n}$, the KL-divergence is usually estimated by estimating $d \mathbb{P} / d \mu$ and $d \mathbb{Q} / d \mu$ individually, using either the Parzen window estimate [2], or the nearest neighbor based estimate [10]. Following the Parzen type approach, $\mathrm{d} \mathbb{P} / \mathrm{d} \mu$ can be estimated as $1 / n \sum_{i=1}^{n} p\left(x, x_{i}\right)$ where $p(x, y)=p(x-y)$ is a Parzen kernel. This approach, however, cannot be readily extended to a more general space since, the space under consideration might not have a Parzen type kernel, e.g. a non-negative function $p(x, y): \mathcal{X} \times \mathcal{X} \rightarrow \mathbb{R}$ that at least satisfies $\int p(x, y) \mathrm{d} \mu(x)=1$. The nearest neighbor based approach, on the other hand, approximates the Radon-Nikodym derivative at a point $x$ as $\mathbb{P}\left(A_{x}\right) / \mu\left(A_{x}\right)$ where $A_{x}$ denotes a neighborhood of $x$, assuming that it is constant in this neighborhood. However, this approach is feasible when $\mu\left(A_{x}\right)$ is known e.g. in $\mathcal{X}=\mathbb{R}^{n}$, where $\mu$ is always considered to be the Lebesgue measure. In a more general space, however, such simple measure might not be available i.e. $\mu\left(A_{x}\right)$ might be difficult to estimate for an arbitrary $A_{x}$.

Recently, there have been some exploratory studies on estimating the Radon-Nikodym derivative $d \mathbb{P} / \mathrm{d} \mathbb{Q}$ directly using strictly positive definite kernels without estimating $\mathrm{dP} / \mathrm{d} \mu$ and $\mathrm{d} \mathbb{Q} / \mathrm{d} \mu$ individually [8]. Although this method has been extended to a more general space, designing an appropriate kernel on the space of spike trains still remains an open issue [6]. On the other hand, metrics on the space of trains are relatively well studied in the literature [9]. Therefore, we consider a nearest neighbor based approach to estimate the Radon-Nikodym derivative $\mathrm{dP} / \mathrm{d} \mathbb{Q}$ directly by assuming that the underlying space $\mathcal{X}$ is a metric space, since the assumption of metric space allows us to define a neighborhood in terms of the associated metric. However, notice that the metric of the space is not unique, and therefore, the proposed approach depends on the choice of the metric. We will address this issue in detail later.
Let $A_{x_{i}}$ denote a neighborhood around $x_{i}$ where we assume that the Radon-Nikodym derivative $d \mathbb{P} / d \mathbb{Q}$ is constant. Then,

$$
\begin{gathered}
\mathbb{P}\left(A_{x_{i}}\right)=\int_{A_{x_{i}}} \frac{\mathrm{d} \mathbb{P}}{\mathrm{d} \mathbb{Q}}(x) \mathrm{d} \mathbb{Q}(x)=\frac{\mathrm{d} \mathbb{P}}{\mathrm{d} \mathbb{Q}}\left(x_{i}\right) \mathbb{Q}\left(A_{x_{i}}\right) \\
\Rightarrow \frac{\mathrm{d} \mathbb{P}}{\mathrm{d} \mathbb{Q}}\left(x_{i}\right)=\frac{\mathbb{P}\left(A_{x_{i}}\right)}{\mathbb{Q}\left(A_{x_{i}}\right)} .
\end{gathered}
$$

Now, the individual probabilities in the numerator and the denominator can be estimated from the empirical probability laws, i.e. $\hat{\mathbb{P}}\left(A_{x_{i}}\right)=k_{x_{i}} / n$ and $\hat{\mathbb{Q}}\left(A_{x_{i}}\right)=l_{x_{i}} / m$ where $k_{x_{i}}$ and $l_{x_{i}}$ are the number of realizations from $X$ and $Y$ that fall in the neighborhood $A_{x_{i}}$.

However, this approach suffers when $\mathbb{P} \nless \mathbb{Q}$ for example when the $\mathbb{P}$ and $\mathbb{Q}$ have disjoint supports, since then $l_{x_{i}}$ could be zero. To avoid this situation, we work with the JensenShannon (JS) divergence instead i.e.

$$
\mathbb{D}_{J S}=\left(\mathbb{D}_{K L}(\mathbb{P}, \xi)+\mathbb{D}_{K L}(\mathbb{Q}, \xi)\right) / 2
$$

where $\xi=(\mathbb{P}+\mathbb{Q}) / 2$. It is evident that $\hat{\xi}\left(A_{x_{i}}\right)=\left(k_{x_{i}} / n+\right.$ $\left.l_{x_{i}} / m\right) / 2>0$. Therefore, the JS-divergence can be estimated as

$$
\begin{aligned}
\hat{\mathbb{D}}_{J S}(\mathbb{P}, \mathbb{Q})= & \frac{1}{2 n} \sum_{i=1}^{n} \log \frac{2 k_{x_{i}} / n}{k_{x_{i}} / n+l_{x_{i}} / m} \\
& +\frac{1}{2 m} \sum_{i=1}^{m} \log \frac{2 k_{y_{i}} / m}{k_{y_{i}} / m+l_{y_{i}} / n}
\end{aligned}
$$

where $k_{y_{i}}$ and $l_{y_{i}}$ are the number of realizations from $Y$ and $X$ that fall in the neighborhood $A_{y_{i}}$ around $y_{i}$.

It still remains an open issue, how to choose the appropriate neighborhood. Exploiting the fact that $\mathcal{X}$ is a metric space, we choose the neighborhood around a sample $x$ to be the sphere of radius $\delta\left(x, x^{(k)}\right)$ where $x^{(k)}$ denotes the $k$-th neighbor of $x$. However, this approach relies on the choice of the metric. To elaborate this issue, let us consider the trivial metric $\delta(x, y)=0$ if $x=y$, or 1 otherwise. Using this metric, for any $k, k_{x_{i}}=n$ and $l_{x_{i}}=m$, and therefore, $\hat{\mathbb{D}}_{J S}=0$, irrespective of the allocation of the realizations. Therefore, it is evident that the proposed approach requires the associated metric to satisfy some regulatory conditions, such as avoiding equivalence classes, i.e. sets of distinct points where the distance between two points is zero. However, the true extent of the effect of the metric on the proposed approach still remains to established. In the simulations, we explore different metrics, and discuss the importance of the metric in more detail. Finally, the size of the neighborhood $k$ is a free parameter, and needs to be chosen by the user. Notice that if the samples $x_{i}$ 's and $y_{i}$ 's are all distinct, then $k_{x}=k$. However, if there are repetitions among the samples, then $k_{x}$ might not always be $k$. In the simulation section, we use $k=\sqrt{\min (n, m)}$. 

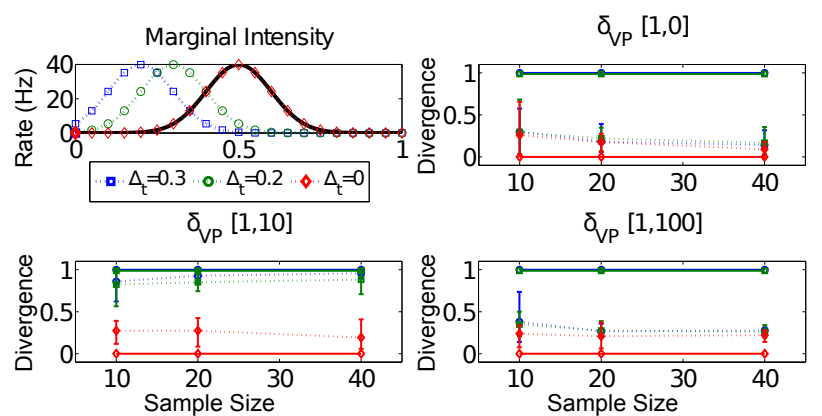

Fig. 1. Estimated divergence between two inhomogeneous Poisson processes with same intensity functions, i.e. Gaussian shaped with $\sigma=0.1 \mathrm{~s}$, but shifted in time by $\Delta_{t} \mathrm{~s}$. Dotted line shows the estimated divergence, and solid line shows the actual divergence computed by Monte Carlo integration. Error bars are plotted over 10 trials.

\section{SIMULATION}

In this section, we apply the proposed estimator in assessing divergence between two point processes. Since the proposed approach depends on the choice of metric, we explore a family of metrics $\delta_{V P}[p, q]$ characterized by parameters $p$ and $q$ [1]. Given two spike trains $\left\{x_{i}\right\}_{i=1}^{n}$ and $\left\{y_{j}\right\}_{j=1}^{m}, \delta_{V P}[p, q]$ measures the minimum cost of matching one spike train with the other by a set of elementary matching operations: adding/deleting a spike at cost 1 or moving a spike in time at cost $q$ per second i.e.

$$
\begin{aligned}
\delta_{V P}[p, q](x, y)=\min _{M}\left[\sum_{\left(x_{i}, y_{j}\right) \in M} q^{p}\left|x_{i}-y_{j}\right|^{p}\right. & \\
& +(m+n-2|M|)]^{1 / p}
\end{aligned}
$$

where $M$ denotes an entire set of matching operations. For simplicity, we choose $p=1$, and vary $q$ which is equivalent to using the metric proposed by [9]. Notice that for $q=0$ or $q \rightarrow \infty$, the metric only depends on the spike counts. Since in practice the number of recorded trials is often around 30, we only use a maximum of $m=n=40$ realizations in our simulations.

Effect of metric To demonstrate the effect of the metric on the estimated divergence value, we generate samples from two inhomogeneous Poisson processes with different mean intensity functions as described in Figure 1. Both intensity functions are shaped like Gaussian distributions with equal variance but with different means. We compare the actual and the estimated divergence values over different deviations of the means. As expected, we observe that for $q=0$ and $q=100$ the estimated values are very different than the actual values, and are close to 0 , whereas for $q=10$ they tend to approach in the vicinity of the actual values.
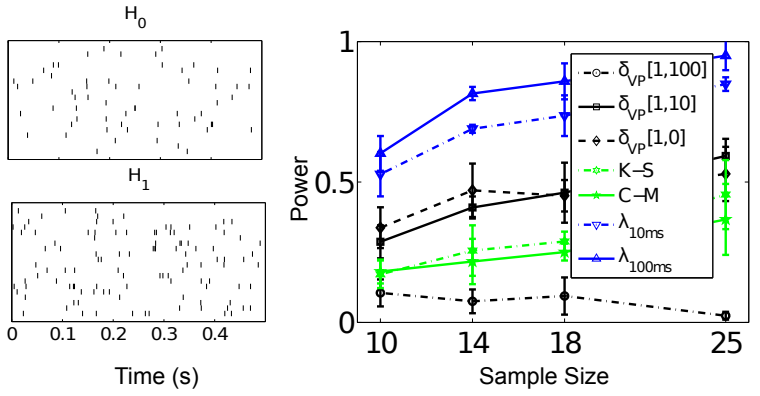

Fig. 2. (Left) Homogeneous Poisson processes: $\mathrm{H}_{0}$ has mean rate 3 spikes/s and $\mathrm{H}_{1}$ has mean rate 5 spikes/s. (Right) Power results. Error bars are plotted over 5 trials.

Hypothesis testing We apply the proposed method in several two-sample problems using point process models. Given two sets of spike trains, the two-sample problem addresses whether they follow the same probability law. To answer this question, we perform a surrogate test to find the appropriate rejection threshold, and reject the hypothesis, that the underlying point processes are the same, if the estimated divergence is above this threshold. Estimating this threshold is necessary since we have seen, in the previous example, that the estimated divergence value usually has bias in finite sample size. We set the size of the test to 0.05 . We compare the performance of the proposed approach against the KS and CM tests as proposed in [7], and the dissimilarity measure $\lambda_{L 2}$, that measures the $L_{2}$ distance between the smoothed time histograms of the spike trains. Both KS and CM are parameter free, whereas $\lambda_{L 2}$ requires choosing an appropriate smoothing kernel and the associated smoothing parameter. We use the IOCANE toolbox (http://code.google.com/p/iocane/) for these methods.

Homogeneous Poisson processes We begin by comparing two homogeneous Poisson processes of different rates (see Figure 2). Since the two processes differ in their marginal intensity functions, $\lambda_{L 2}$ can easily distinguish between them. However, since these two processes exhibit wide count distributions, both KS and CM fail to achieve good separation. The proposed approach, on the other hand, outperforms both KS and CM for $q=0$ and $q=10$, but fails for $q=100$. While with $q=10$ the method works as expected, the performance of $q=0$ is attributed to the fact that the processes can be separated in terms of the difference in their spike counts. On the other hand, $q=100$ i.e. $q \rightarrow \infty$ tends to compare two spike trains by their combined spike counts, and this does not reveal the difference between the underlying point processes.

Next, we consider two renewal processes with same mean firing rate, but gamma distributed interspike intervals with different shape parameters (see Figure 3). This problem has been addressed in [7], and it has been shown that both KS and $\mathrm{CM}$ outperform other methods. Since both the processes have the same marginal intensity function, $\lambda_{L 2}$ should fail to 

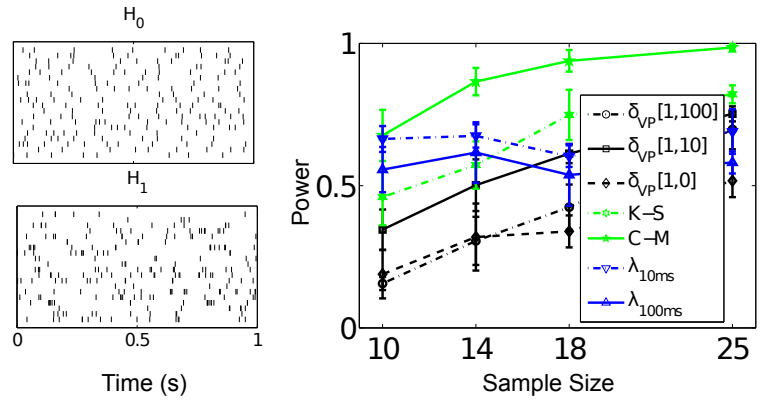

Fig. 3. (Left) Renewal processes: mean rate is 10 spikes/s, and interspike intervals are Gamma distributed; $\mathrm{H}_{0}$ has shape parameter 3 and $\mathrm{H}_{1}$ has shape parameter 0.5. (Right) Power results. Error bars are plotted over 5 trials.

distinguish between these two processes. However, a higher variability in the marginal intensity function of the alternate set of spike trains helps discriminate the processes. But, this performance does not improve with the sample size. The proposed method performs well for all $q$ 's, but does not match the power of KS and CM. The performance of $q=0$ and $q=10$ can be explained as in the previous example, whereas the performance of $q=100$ can be attributed to the increased temporal resolution which is appropriate for favoring the more consistent firing of the spike trains from the null hypothesis. Inhomogeneous Poisson processes Finally, we consider two inhomogeneous Poisson processes where one process has a Gaussian shaped marginal intensity function, and the other process toggles between two Gaussian shaped marginal intensity functions with different means (see Figure 4). Since the marginal intensity functions are very similar, $\lambda_{L 2}$ fails to correctly separate these two point processes, whereas KS and $\mathrm{CM}$ suffer from estimation issues. On the other hand, both $q=0$ and $q=100$ also fail to distinguish between these two point processes due to lack of difference in count distributions. However, $q=10$ provides promising performance as expected.

\section{DISCUSSION}

In this paper, we have explored the estimation of JensenShannon divergence between two point processes by exploiting the fact the space of spike trains can be treated as a metric space. The proposed approach does not stratify the space of spike trains, and therefore allows pairwise interaction between the realizations irrespective of their spike counts. This in turn allows the proposed method to be more robust in the situations where the count distributions of the point processes are wide.

The proposed method requires selecting two parameters, namely the neighborhood size, and an appropriate metric. We have shown that the choice of metric is crucial for the better performance of the proposed method. But, given a suitable
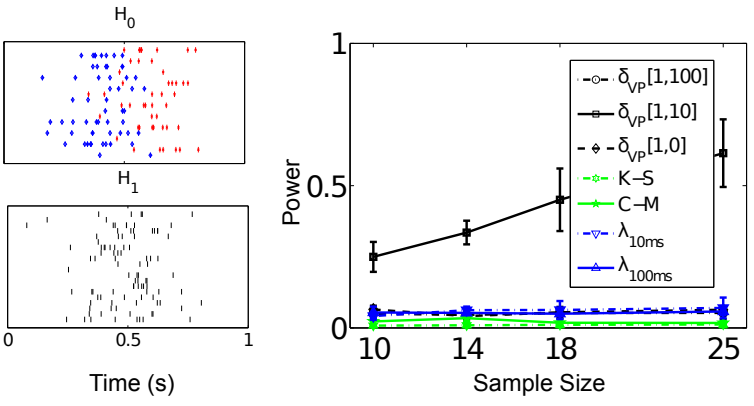

Fig. 4. (Left) Inhomogeneous Poisson processes: $\mathrm{H}_{0}$ is a process which toggles between different inhomogeneous processes whose intensity functions are Gaussians with mean $0.4 \mathrm{~s}$ or $0.6 \mathrm{~s}$ and $\sigma_{0}=$ $0.1 \mathrm{~s} . \mathrm{H}_{1}$ has a Gaussian shaped intensity function with mean $0.5 \mathrm{~s}$ and $\sigma_{1}^{2}=2 \sigma_{0}^{2}$. (Right) Power results. Error bars are plotted over 5 trials.

metric, the proposed approach provide robust performance over different statistical nature of the underlying processes, where other available methods fail. However, the choice of neighborhood size remains to be explored in its entirety.

The performance of the hypothesis testing can also be used to select the best metric that, in essence, reveals the most information about the two point processes. This idea is very similar to the approach considered by [9], but it avoids explicit clustering of the realizations.

\section{REFERENCES}

[1] A. J. Dubbs, B. A. Seiler, and M. O. Magnasco. A fast $\mathcal{L}_{p}$ spike alignment metric. Neural Comput., 22(11):2785-2808, 2010.

[2] C. Granger and J. L. Lin. Using the mutual information coefficient to identify lags in nonlinear models. J. Time Ser. Anal., 15-4:371 - 384, 1991.

[3] A. Gretton, K. M. Borgwardt, M. J. Rasch, B. Schölkopf, and Alexander J. Smola. A kernel method for the two-sample problem. CoRR, abs/0805.2368, 2008.

[4] M. Hein and O. Bousquet. Hilbertian metrics and positive definite kernels on probability measures. In AISTATS 10, pages 136-143, 2005.

[5] I. Park and J. C. Príncipe. Quantification of inter-trial non-stationarity in spike trains from periodically stimulated neural cultures. In ICASSP, 2010. Special session on Multivariate Analysis of Brain Signals: Methods and Applications.

[6] I. Park, S. Seth, M. Rao, and J.C. Príncipe. Estimation of symmetric chi-square divergence for point processes. ICASSP, 2011 (In press).

[7] S. Seth, I. Park, A. Brockmeier, M. Semework, J. Choi, J. Francis, and J. C. Príncipe. A novel family of non-parametric cumulative based divergences for point processes. NIPS 24, pages 2119-2127, 2010.

[8] M. Sugiyama, S. Nakajima, H. Kashima, P. V. Buenau, and M. Kawanabe. Direct importance estimation with model selection and its application to covariate shift adaptation. NIPS 22, pages 1433-1440, 2008.

[9] J. D. Victor and K. P. Purpura. Metric-space analysis of spike trains: theory, algorithms and application. Network: Comput. Neural Syst, 8(2):127-164, 1997.

[10] Q. Wang, S. R. Kulkarni, and S. Verdú. Divergence estimation for multidimensional densities via k-nearest-neighbor distances. IEEE Trans. Inf. Theor., 55(5):2392-2405, 2009. 